\title{
Avances en la Predicción de Propiedades Físicas, Físico-Químicas y de Transporte de Líquidos lónicos
}

\author{
José O. Valderrama* ${ }^{1,2}$ y Roberto E. Rojas ${ }^{3}$ \\ (1)Univ. de La Serena, Fac. de Ingeniería, Dpto. de Ing. Mecánica., Casilla 554, La Serena-Chile \\ (2) Centro de Información Tecnológica (CIT), Casilla 724, La Serena-Chile \\ (3) Univ. de La Serena, Fac. de Ciencias, Dpto. de Química, Casilla 554, La Serena-Chile
}

\begin{abstract}
Resumen
Se presenta y discute los avances del grupo de investigación de la Universidad de La Serena sobre la estimación de las propiedades físicas, fisicoquímicas y de transporte de líquidos iónicos (LIs). Aunque se ha sostenido que los métodos usuales que se aplican a fluidos orgánicos no pueden ser aplicados a los LIs y que se requiere un nuevo formalismo que explique el comportamiento especialmente diferente de los LIs, el grupo de trabajo de La Serena ha demostrado que esto no es correcto. Este trabajo presenta los resultados de varios métodos tradicionales para estimar las propiedades de fluidos que han sido aplicados a los LIs; en particular, técnicas de contribución de grupos, correlaciones generalizadas, correlaciones empíricas, y redes neuronales. De esta forma se han correlacionado y predicho hasta la fecha las propiedades críticas, la presión de vapor, la densidad y la viscosidad de líquidos iónicos.
\end{abstract}

Palabras clave: líquidos iónicos, contribución de grupos, propiedades críticas, presión de vapor, viscosidad

\section{Advances in the Prediction of Physical, Physicochemical and Transport Properties of Ionic Liquids}

\begin{abstract}
The advances done by the research group in La Serena-Chile on the estimation of several physical, physicochemical, and transport properties of ionic liquids (ILS) are presented and analyzed. Although it has been mentioned in the literature that the methods usually applicable to organic fluids cannot be used to estimate the properties of ILs and a new formalism to explain the especial behavior of ILs should be formulated, the group in La Serena has demonstrated that this is not correct. This work presents the results of applying several traditional methods for estimating some properties of ILs; in particular, group contribution techniques, generalized correlation, empirical correlations, and neural networks have been employed. Until now, the critical properties, the vapor pressure, the density and the viscosity of ILs have been correlated and predicted.
\end{abstract}

Keywords: ionic liquids, group contribution, critical properties, vapor pressure, viscosity 


\section{INTRODUCCIÓN}

En años recientes, los líquidos iónicos (LIs) han generado especial atención por sus potenciales usos como solventes medioambientalmente benignos y como posibles reemplazantes de los tradicionales solventes orgánicos volátiles. Son definidos como fluidos formados solamente por iones con temperaturas de fusión inferiores a $100^{\circ} \mathrm{C}$. A temperatura ambiente, también se conocen como líquidos iónicos y permanecen líquidos en un amplio intervalo de temperatura. Ningún solvente molecular, excepto algunos polímeros, puede igualar el intervalo líquido de estos compuestos, que es del orden de $300^{\circ} \mathrm{C}$. Estos líquidos han sido conocidos desde comienzos del siglo pasado, siendo el nitrato de etilamonio, $\mathrm{EtNH}_{3}-\mathrm{NO}_{3}$, el primer líquido iónico descubierto, en el año 1914 (Rogers y Seddon, 2002). El interés de parte de los científicos y la industria por los líquidos iónicos ha ido creciendo rápidamente en todo el mundo, demostrado por el acelerado número de publicaciones y patentes sobre los líquidos iónicos. En el año 1990, los trabajos no superaban las 100 publicaciones llegando a más de 1000 artículos publicados en el año 2004, y a casi 2500, el año 2008 (ISI, 2009), además de más de 200 libros publicados en los últimos diez años (GB, 2009). El interés por usar líquidos iónicos como disolventes en diferentes procesos químicos se debe a que estos compuestos son térmicamente estables, tienen una elevada polaridad, permanecen inalterados cuando se mezclan con diferentes compuestos orgánicos y catalizadores, tienen una presión de vapor muy pequeña, son líquidos en un amplio intervalo de temperatura y son capaces de ser miscibles e inmiscibles con sustancias orgánicas o inorgánicas. Este conjunto de propiedades permite diseñar sistemas de reacción muy atractivos que pueden resolver los principales inconvenientes de algunos métodos de síntesis empleados actualmente o abordar nuevos procedimientos para fabricar productos (Wasserscheid y Welton, 2002; Welton, 1999).

A medida que se han ido conociendo las singulares propiedades de los LIs, ha crecido el interés por aplicarlos como medio de reacción en una gran variedad de transformaciones químicas que hasta hace poco sólo podían efectuarse en compuestos orgánicos o en disolventes acuosos. La literatura describe numerosas aplicaciones: como solventes en electroquímica, reacciones enzimáticas y reacciones a elevadas temperaturas; en separación de metales, absorción de gases $\left(\mathrm{CO}_{2}\right)$ y como agente desecante para gases; como líquido térmico para conducir y almacenar calor; como aditivo en la fabricación de plásticos, en combustibles y lubricantes. Además, son una alternativa reciclable en solventes apróticos (en solución pueden donar átomos de hidrógeno y/o formar puentes de hidrógeno), catalizadores en síntesis orgánica y organometálica y para polimerización de radicales, y algunos líquidos iónicos pueden ser usados como biocatalizadores con grandes ventajas comparados con los solventes orgánicos convencionales (Wypych, 2001). La Fig. 1 muestra los diversos usos y aplicaciones de los líquidos iónicos en distintas áreas de la ciencia y de la tecnología.

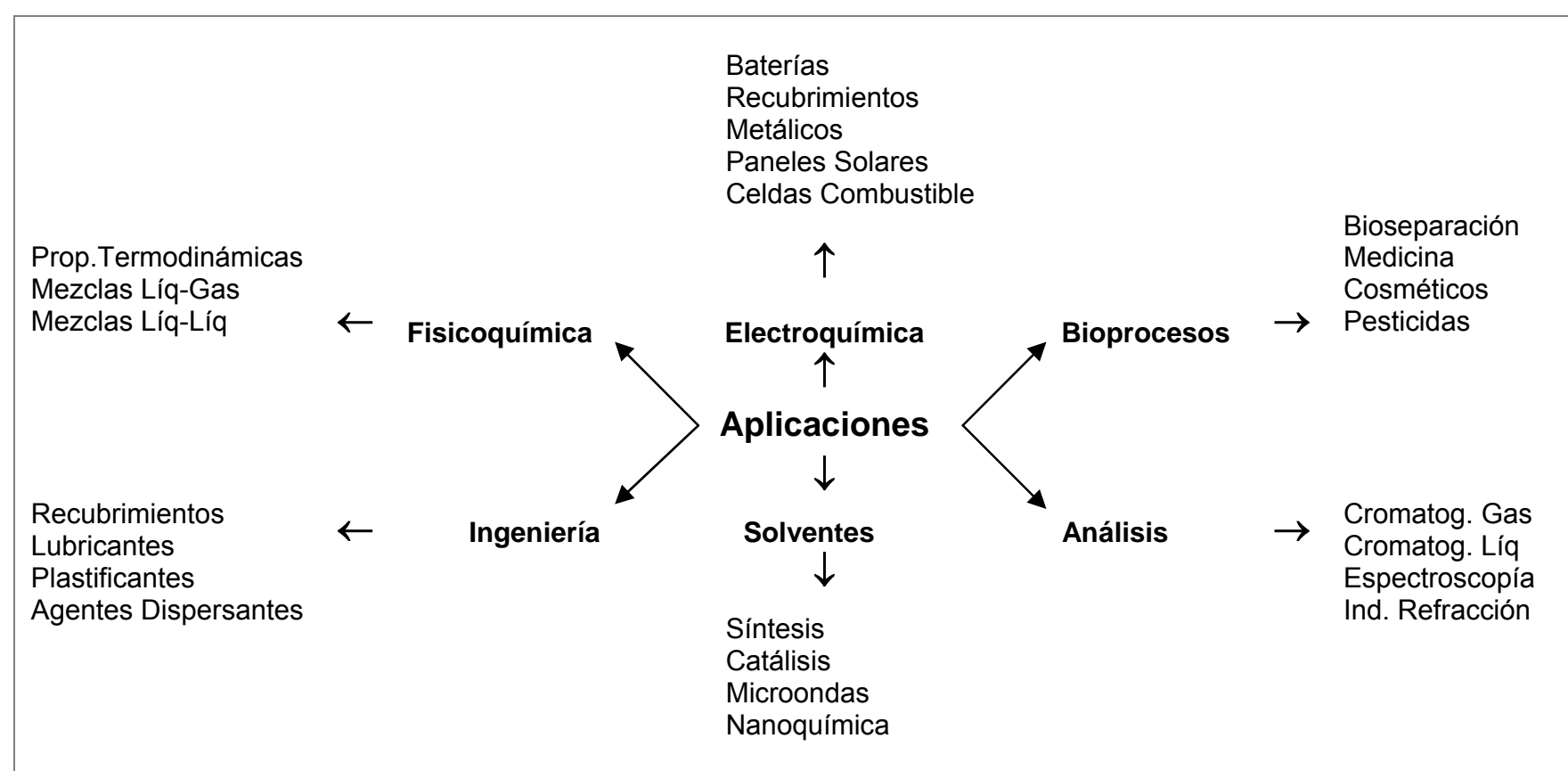

Fig.1: Usos y aplicaciones de los líquidos iónicos en diversos sectores industriales. 
En el tema de la estimación de propiedades, se ha postulado que los métodos usuales que se aplican a fluidos orgánicos no pueden ser aplicados a los LIs y que se requiere un nuevo formalismo que explique el comportamiento especialmente diferente de los LIs (Wypych, 2001). Sin embargo, el grupo de La Serena ha mostrado que los métodos desarrollados para los fluidos moleculares pueden ser extendidos para correlacionar y predecir las propiedades de los LIs. Todo esto ha motivado el trabajo del grupo de La Serena, en particular en la estimación de las propiedades necesarias de los LIs en la simulación y diseño de procesos.

En este artículo se presentan y discuten los avances del grupo de investigación de la Universidad de La Serena sobre la estimación de las propiedades físicas, fisicoquímicas y de transporte de los LIs. Se muestran los resultados de varios métodos tradicionales para estimar sus propiedades, en particular, las técnicas de contribución de grupos, correlación generalizada, correlaciones empíricas, y redes neuronales para predecir varias propiedades de los LIs, tales como propiedades críticas, presión de vapor, densidad, y viscosidad, entre otras. Los principales avances del Grupo de La Serena-Chile que se describen más adelante se resumen en las siguientes aplicaciones: i) método de contribución de grupos denominado Lydersen-Joback-Modificado (Alvarez y Valderrama, 2004) para correlacionar y predecir varias propiedades, incluido el método más completo disponible para estimar las propiedades críticas de los LIs (Valderrama y Robles 2007a; 2007b; Valderrama et al., 2008a; Valderrama y Rojas, 2009); ii) método generalizado para estimar la densidad de Lls (Valderrama y Zarricueta, 2009); correlaciones para la presión de vapor (Valderrama y Zarricueta, c2009) iv) densidad y viscosidad de LIs usando redes neuronales (Valderrama et al. 2009; Valderrama et al., c2008). Actualmente se trabaja en la definición de descriptores moleculares que permitan en forma simple correlacionar y predecir varias propiedades en las que la especial estructura molecular de los LIs tiene especial ingerencia.

\section{RESULTADOS}

El grupo de La Serena ha realizado estudios de la correlación y predicción de varias propiedades físicas, fisicoquímicas y de transporte, como también estudios con mezclas que contienen líquidos iónicos. La Tabla 1 resume estos estudios, algunos ya realizados y otros en progreso, y se indica la referencia de aquellos que han sido publicados en revistas de corriente principal y presentados en congresos.

Tabla 1: Avances en el estudio de propiedades de los LIs en el grupo de La Serena

\begin{tabular}{|c|c|c|}
\hline Propiedades & Publicaciones & Congresos \\
\hline Varias & ------------ & $\begin{array}{l}\text { Valderrama et al. Asunción-Paraguay (c2007) } \\
\text { Valderrama, Chiclayo-Perú (c2008) } \\
\text { Valderrama, San Rafael-Argentina (c2009) } \\
\text { Valderrama, Aveiro-Portugal (c2009a; c2009b) }\end{array}$ \\
\hline $\begin{array}{l}\text { Propiedades } \\
\text { Críticas }\end{array}$ & $\begin{array}{l}\text { Valderrama y Robles (2007a; 2007b) } \\
\text { Valderrama et al. (2008a) } \\
\text { Valderrama y Rojas (2009) }\end{array}$ & Valderrama y Lazzus, Frankfurt-Alemania (c2008) \\
\hline Densidad & $\begin{array}{l}\text { Valderrama y Zarricueta (2009) } \\
\text { Valderrama et al. (2009) }\end{array}$ & $\begin{array}{l}\text { Valderrama y Reátegui, Chiclayo-Perú (c2009) } \\
\text { Valderrama et al., Frankfurt-Alemania (c2008) }\end{array}$ \\
\hline $\begin{array}{l}\text { Presión de } \\
\text { Vapor }\end{array}$ & ------------ & $\begin{array}{l}\text { Valderrama y Lazzus, Frankfurt-Alemania (c2008) } \\
\text { Valderrama y Zarricueta, San Rafael-Argentina (c2009) }\end{array}$ \\
\hline Viscosidad & ---------- & Valderrama et al., San Rafael-Argentina (c2009) \\
\hline $\begin{array}{l}\text { Equilibrio } \\
\text { Líquido-Gas }\end{array}$ & Valderrama et al. (2008b) & Valderrama, Aveiro-Portugal (c2009) \\
\hline
\end{tabular}




\section{Propiedades Críticas}

En el grupo de La Serena se ha desarrollado un método de contribución de grupos denominado método Modificado Lydersen-Joback-Reid y se han definido los grupos necesarios para predecir las propiedades críticas de los LIs (Valderrama y Robles, 2007a y 2007b; Valderrama et al., 2008a; Valderrama y Rojas, 2009). La Tabla 2 muestra los grupos y los valores de las contribuciones, la Tabla 3 muestra las ecuaciones que describen el método de contribución de grupos y la Tabla 4 presenta una selección de valores de propiedades críticas de algunos LIs.

Tabla 2: Grupos incluidos en el Método Lydersen-Joback-Reid para LIs (Valderrama y Rojas, 2009)

\begin{tabular}{|c|c|c|c|c|c|}
\hline Grupos & $M_{i}$ & $\Delta T_{c}$ & $\Delta P_{c}$ & $\Delta V_{c}$ & $\Delta T_{b}$ \\
\hline & & Sin anillos & & & \\
\hline$-\mathrm{CH}_{3}$ & 15.035 & 0.0275 & 0.3031 & 66.81 & 23.58 \\
\hline$-\mathrm{CH}_{2-}^{-}$ & 14.027 & 0.0159 & 0.2165 & 57.11 & 22.88 \\
\hline$>\mathrm{CH}_{-}$ & 13.019 & 0.0002 & 0.114 & 45.7 & 21.74 \\
\hline$>\mathrm{C}<$ & 12.011 & -0.0206 & 0.0539 & 21.78 & 18.25 \\
\hline$=\mathrm{CH}_{2}$ & 14.027 & 0.017 & 0.2493 & 60.37 & 18.18 \\
\hline$=\mathrm{CH}-$ & 13.019 & 0.0182 & 0.1866 & 49.92 & 24.96 \\
\hline$=\mathrm{C}<$ & 12.011 & -0.0003 & 0.0832 & 34.9 & 24.14 \\
\hline$=\mathrm{C}=$ & 12.011 & -0.0029 & 0.0934 & 33.85 & 26.15 \\
\hline$\equiv \mathrm{CH}$ & 13.019 & 0.0078 & 0.1429 & 43.97 & --- \\
\hline -COO- & 12.011 & 0.0078 & 0.1429 & 43.97 & --- \\
\hline$-\mathrm{OH}$ & 17.008 & 0.0723 & 0.1343 & 30.4 & 92.88 \\
\hline$-\mathrm{O}-$ & 16.000 & 0.0051 & 0.13 & 15.61 & 22.42 \\
\hline$>\mathrm{C}=\mathrm{O}$ & 28.011 & 0.0247 & 0.2341 & 69.76 & 94.97 \\
\hline$-\mathrm{CHO}$ & 29.019 & 0.0294 & 0.3128 & 77.46 & 72.24 \\
\hline$-\mathrm{COOH}$ & 45.018 & 0.0853 & 0.4537 & 88.6 & 169.06 \\
\hline -COO- & 44.010 & 0.0377 & 0.4139 & 84.76 & 81.10 \\
\hline$-\mathrm{HCOO}-$ & 45.018 & 0.036 & 0.4752 & 97.77 & --- \\
\hline$=0$ (cualquier otro) & 16.000 & 0.0273 & 0.2042 & 44.03 & -10.50 \\
\hline$-\mathrm{NH}_{2}$ & 16.023 & 0.0364 & 0.1692 & 49.1 & 73.23 \\
\hline$-\mathrm{NH}_{3}$ & 17.031 & 0.0364 & 0.1692 & 49.1 & 73.23 \\
\hline$-\mathrm{NH}-$ & 15.015 & 0.0119 & 0.0322 & 78.96 & 50.17 \\
\hline$>\mathrm{N}-$ & 14.007 & -0.0028 & 0.0304 & 26.7 & 11.74 \\
\hline$=\mathrm{N}-$ & 14.007 & 0.0172 & 0.1541 & 45.54 & 74.60 \\
\hline$-\mathrm{CN}$ & 26.018 & 0.0506 & 0.3697 & 89.32 & 125.66 \\
\hline$-\mathrm{NO}_{2}$ & 46.006 & 0.0448 & 0.4529 & 123.62 & 152.54 \\
\hline$-F$ & 18.999 & 0.0228 & 0.2912 & 31.47 & -0.03 \\
\hline$-\mathrm{Cl}$ & 35.453 & 0.0188 & 0.3738 & 62.08 & 38.13 \\
\hline$-B r$ & 79.904 & 0.0124 & 0.5799 & 76.6 & 66.86 \\
\hline-1 & 126.905 & 0.0148 & 0.9174 & 100.79 & 93.84 \\
\hline$-\mathrm{P}$ & 30.974 & -0.0084 & 0.1776 & 67.01 & 34.86 \\
\hline$-B$ & 10.811 & 0.0352 & 0.0348 & 22.45 & -24.56 \\
\hline -S- & 32.066 & 0.0006 & 0.6901 & 184.67 & 117.52 \\
\hline \multirow[t]{2}{*}{$\mathrm{O}=\mathrm{S}=\mathrm{O}$} & 64.065 & -0.0563 & -0.0606 & 112.19 & 147.24 \\
\hline & & Con anillos & & & \\
\hline$-\mathrm{CH}_{2-}^{-}$ & 14.027 & 0.0116 & 0.1982 & 51.64 & 27.15 \\
\hline$>\mathrm{CH}-$ & 13.019 & 0.0081 & 0.1773 & 30.56 & 21.78 \\
\hline$=\mathrm{CH}-$ & 13.019 & 0.0114 & 0.1693 & 42.55 & 26.73 \\
\hline$>\mathrm{C}<$ & 12.011 & -0.018 & 0.0139 & 17.62 & 21.32 \\
\hline$=\mathrm{C}<$ & 12.011 & 0.0051 & 0.0955 & 31.28 & 31.01 \\
\hline$-\mathrm{O}-$ & 16.000 & 0.0138 & 0.1371 & 17.41 & 31.22 \\
\hline -OH (fenoles) & 17.008 & 0.0291 & 0.0493 & -17.44 & 76.34 \\
\hline$>\mathrm{C}=\mathrm{O}$ & 28.011 & 0.0343 & 0.2751 & 59.32 & 94.97 \\
\hline$-\mathrm{NH}-$ & 15.015 & 0.0244 & 0.0724 & 27.61 & 52.82 \\
\hline$[>\mathrm{N}<]^{+}$ & 14.007 & 0.0063 & 0.0538 & 25.17 & 68.16 \\
\hline$[>\mathrm{N}=]^{+}$ & 14.007 & -0.0011 & 0.0559 & 42.15 & 57.55 \\
\hline
\end{tabular}


Tabla 3: Método Lydersen-Joback-Reid (Alvarez y Valderrama, 2004)

\begin{tabular}{|l|l|}
\hline Modelo & Constantes \\
\hline $\mathrm{T}_{\mathrm{b}}=198.2+\sum \mathrm{n} \Delta \mathrm{T}_{\mathrm{b}}$ & $\mathrm{A}=0.5703$ \\
\hline $\mathrm{T}_{\mathrm{c}}=\mathrm{T}_{\mathrm{b}} /\left\{\mathrm{A}+\mathrm{B} \sum \mathrm{n} \Delta \mathrm{T}-\sum(\mathrm{n} \Delta \mathrm{T})^{2}\right\}$ & $\mathrm{B}=1.0121$ \\
\hline $\mathrm{P}_{\mathrm{c}}=\mathrm{M} /\left\{\mathrm{C}+\sum \mathrm{n} \Delta \mathrm{P}\right\}^{2}$ & $\mathrm{C}=0.2573$ \\
\hline $\mathrm{V}_{\mathrm{c}}=\mathrm{E}+\sum \mathrm{n} \Delta V$ & $\mathrm{E}=6.75$ \\
\hline
\end{tabular}

Tabla 4: Propiedades críticas de algunos líquidos iónicos (Valderrama y Rojas, 2009)

\begin{tabular}{|c|c|c|c|c|c|c|}
\hline$N^{\circ}$ & $L I$ & Nombre IUPAC & $T_{b}(K)$ & $T_{C}(K)$ & $\begin{array}{c}P_{C} \\
\text { (bar) }\end{array}$ & $\begin{array}{c}V_{C} \\
\left(\mathrm{~cm}^{3} / \mathrm{mol}\right)\end{array}$ \\
\hline 1 & [bmim] [TFES] & $\begin{array}{l}\text { 1-butyl-3-methylimidazolium 1,1,2,2- } \\
\text { tetrafluoroethanosulfonate }\end{array}$ & 827.8 & 729.4 & 1030.5 & 25.7 \\
\hline 2 & [emim] [TFES] & $\begin{array}{l}\text { 1-ethyl-3-methylimidazolium 1,1,2,2- } \\
\text { tetrafluoroethanosulfonate }\end{array}$ & 713.6 & 683.7 & 998.2 & 30.4 \\
\hline 3 & [hpmim] [TFES] & $\begin{array}{l}\text { 1-heptyl-3-methylimidazolium 1,1,2,2- } \\
\text { tetrafluoroethanosulfonate }\end{array}$ & 999.2 & 798.1 & 1080.8 & 20.7 \\
\hline 4 & [emim] [BEI] & $\begin{array}{l}\text { 1-ethyl-3-methylimidazolium } \\
\text { bis(pentafluoroethylsulfonyl)imide }\end{array}$ & 1045.4 & 853.1 & 1231.4 & 21.9 \\
\hline 5 & [bmim] [BEI] & $\begin{array}{l}\text { 1-butyl-3-methylimidazolium } \\
\text { bis(pentafluoroethylsulfonyl)imide }\end{array}$ & 1159.6 & 898.8 & 1257.1 & 19.5 \\
\hline 6 & [4MOPY] [BEI] & $\begin{array}{l}\text { 4-methyl-n-octylpyridinium } \\
\text { bis(pentafluoroethylsulfonyl)imide }\end{array}$ & 1436.7 & 979.9 & 1291.4 & 15.0 \\
\hline 7 & [NH221] [BEI] & $\begin{array}{l}\text { diethylmethyl(quaternary)ammonium } \\
\text { bis(pentafluoroethylsulfonyl)imide }\end{array}$ & 1053.3 & 743.8 & 1056.3 & 21.5 \\
\hline 8 & [dmprim] [bti] & $\begin{array}{l}\text { 1,2-dimethyl-3-propylimidazolium } \\
\text { bis[(trifluoromethyl)sulfonyl]imide }\end{array}$ & 988.6 & 867.4 & 1269.7 & 27.5 \\
\hline 9 & [dbim] [bti] & $\begin{array}{l}\text { 1,3-dibutylimidazolium } \\
\text { bis[(trifluoromethyl)sulfonyl]imide }\end{array}$ & 1161.5 & 931.1 & 1305.0 & 22.3 \\
\hline 10 & [E1,3M4I] [bti] & $\begin{array}{l}\text { 1,3-diethyl-4-methylimidazolium } \\
\text { bis[(trifluoromethyl)sulfonyl]imide }\end{array}$ & 988.6 & 867.4 & 1269.7 & 27.5 \\
\hline 11 & [dmim] [bti] & $\begin{array}{l}\text { 1,3-dimethylimidazolium } \\
\text { bis[(trifluoromethyl)sulfonyl]imide }\end{array}$ & 818.8 & 793.8 & 1239.9 & 35.8 \\
\hline 12 & [bmpy] [bti] & $\begin{array}{l}\text { 1-butyl-3-methylpyridinium } \\
\text { bis[(trifluoromethyl)sulfonyl]imide }\end{array}$ & 1038.8 & 852.0 & 1240.5 & 25.5 \\
\hline 13 & [N-epy] [bti] & $\begin{array}{l}\text { 1-ethylpyridinium } \\
\text { bis[(trifluoromethyl)sulfonyl]imide }\end{array}$ & 869.0 & 778.4 & 1207.9 & 32.7 \\
\hline 14 & [hpmim] [bti] & $\begin{array}{l}\text { 1-heptyl-3-methylimidazolium } \\
\text { bis[(trifluoromethyl)sulfonyl]imide }\end{array}$ & 1161.5 & 931.1 & 1305.0 & 22.3 \\
\hline 15 & [nmim] [bti] & $\begin{array}{l}\text { 1-nonyl-3-methylimidazolium } \\
\text { bis[(trifluoromethyl)sulfonyl]imide }\end{array}$ & 1275.7 & 976.8 & 1331.2 & 19.8 \\
\hline 16 & [pmim] [bti] & $\begin{array}{l}\text { 1-pentyl-3-methylimidazolium } \\
\text { bis[(trifluoromethyl)sulfonyl]imide }\end{array}$ & 1047.2 & 885.3 & 1281.1 & 25.6 \\
\hline 17 & [MP3] [bti] & $\begin{array}{l}\text { 1-propyl-2-methylpyrrolidinium } \\
\text { bis([trifluoromethyl)sulfonyl]imide }\end{array}$ & 981.8 & 735.8 & 1096.2 & 27.4 \\
\hline 18 & [prmim] [bti] & $\begin{array}{l}\text { 1-propyl-3-methylimidazolium } \\
\text { bis[(trifluoromethyl)sulfonyl]imide }\end{array}$ & 933.0 & 839.6 & 1259.3 & 30.0 \\
\hline 19 & [prmpy] [bti] & $\begin{array}{l}\text { 3-methyl-1-propylpyridinium } \\
\text { bis[(trifluoromethyl)sulfonyl]imide }\end{array}$ & 981.7 & 829.1 & 1228.9 & 27.5 \\
\hline 20 & [dmeim] [bti] & $\begin{array}{l}\text { 1,2-dimethyl-3-ethylimidazolium } \\
\text { bis[(trifluoromethyl)sulfonyl]imide }\end{array}$ & 931.5 & 844.5 & 1258.9 & 29.8 \\
\hline 21 & [BNM2E] [bti] & $\begin{array}{l}\text { dimethylethylbutylammonium } \\
\text { bis[(trifluoromethyl)sulfonyl]imide }\end{array}$ & 1012.6 & 738.3 & 1054.3 & 24.1 \\
\hline 22 & [eomim] [bti] & $\begin{array}{l}\text { ethoxymethyl-3-methylimidazolium } \\
\text { bis[(trifluoromethyl)sulfonyl]imide }\end{array}$ & 948.6 & 862.0 & 1285.2 & 29.1 \\
\hline
\end{tabular}




\section{Densidad de Líquidos lónicos}

Valderrama y Abu-Shark (1989) desarrollaron una ecuación generalizada para estimar la densidad de líquidos, método que requiere la temperatura de ebullición normal, la masa molecular y las propiedades críticas. La ecuación es:

$\rho_{\mathrm{L}}=\left(\mathrm{MP}_{\mathrm{C}} / \mathrm{RT}_{\mathrm{C}}\right)\left[\left(0.3445 \mathrm{P}_{\mathrm{C}} / \mathrm{RT}_{\mathrm{C}}\right) \mathrm{V}_{\mathrm{C}}^{1.0135}\right]$

$$
-\left[1+\left(1-T_{R}\right)^{2 / 7}\right] /\left[1+\left(1-T_{b R}\right)^{2 / 7}\right]
$$

En esta ecuación, $\rho_{L}$ es la densidad en $\left(\mathrm{gr} / \mathrm{cm}^{3}\right)$, R es la constante universal del gas ideal, $T_{R}$ es la temperatura reducida $\left(T_{R}=T / T_{C}\right)$, y $T_{b R}$ es la temperatura reducida en el punto de ebullición normal $\left(T_{b R}=T_{b} / T_{C}\right)$. Cuando se aplica este modelo a los Lls se obtienen resultados aceptables (Valderrama y Robles, 2007). Sin embargo, mejores resultados se obtuvieron mediante una simplificación y adaptación de este modelo para obtener un modelo lineal general válido para cualquier LI (Valderrama y Zarricueta, 2009).

$$
\begin{aligned}
& \rho=(A / B)+(2 / 7) \cdot\{(A \cdot L n B) / B\} \frac{\left(T-T_{b}\right)}{\left(T_{c}-T_{b}\right)} \\
& A=a+b \cdot M / V_{c} \\
& B=\left(c / V_{c}+d / M\right) \cdot V_{c}^{\delta}
\end{aligned}
$$

Las constantes $\mathrm{a}, \mathrm{b}, \mathrm{c}, \mathrm{d}$ y $\delta$ fueron determinadas usando datos publicados, como se detalla en Valderrama y Zarricueta (2009). Los valores de las constantes son: $a=0.3411, \quad b=2.0443, c=$ 0.5386, $\mathrm{d}=0.0393, \delta=1.0476$. La Tabla 5 muestra una selección de valores de densidad

\begin{tabular}{|c|c|c|c|c|c|c|}
\hline$N^{\circ}$ & Liq. lónico & $\rho(\exp )$ & $\rho(1)$ & $\% \Delta \rho$ & $\rho(2)$ & $\% \Delta \rho$ \\
\hline 1 & {$[\mathrm{P} 11][\mathrm{tsac}]$} & 1.430 & 1.372 & -4.1 & 1.385 & -3.2 \\
\hline 2 & [emim] [tsac] & 1.460 & 1.456 & -0.3 & 1.433 & -1.9 \\
\hline 3 & [TEA] [tsac] & 1.370 & 1.341 & -2.1 & 1.304 & -4.8 \\
\hline 4 & [TMAIA] [tsac] & 1.380 & 1.375 & -0.4 & 1.377 & -0.2 \\
\hline 5 & [TMEA] ]tsac] & 1.400 & 1.387 & -0.9 & 1.401 & 0.1 \\
\hline 6 & [TMiPA] [tsac] & 1.410 & 1.367 & -3.0 & 1.366 & -3.1 \\
\hline 7 & [TMPA] [tsac] & 1.380 & 1.368 & -0.8 & 1.364 & -1.1 \\
\hline 8 & [bmim] [TFES] & 1.324 & 1.302 & -1.7 & 1.313 & -0.8 \\
\hline 9 & [dmim] [TFES] & 1.136 & 1.250 & 10.0 & 1.144 & 0.7 \\
\hline 10 & [emim] [TFES] & 1.502 & 1.345 & -10.5 & 1.387 & -7.6 \\
\hline 11 & [hpmim] [TFES] & 1.274 & 1.267 & -0.6 & 1.232 & -3.3 \\
\hline 12 & [bmim] [HFPS] & 1.409 & 1.391 & -1.3 & 1.366 & -3.1 \\
\hline 13 & {$[4,4,4,14-\mathrm{P}][\mathrm{HFPS}]$} & 1.070 & 1.270 & 18.7 & 1.055 & -1.4 \\
\hline 14 & [bmim] [TPES] & 1.423 & 1.514 & 6.4 & 1.437 & 1.0 \\
\hline 15 & {$[6,6,6,14-\mathrm{P}][\mathrm{TPES}]$} & 1.063 & 1.262 & 18.7 & 1.054 & -0.9 \\
\hline 16 & [bmim] [TTES] & 1.393 & 1.437 & 3.2 & 1.397 & 0.3 \\
\hline 17 & [bmim] [FS] & 1.449 & 1.514 & 4.5 & 1.437 & -0.9 \\
\hline 18 & [TMG] [Ac] & 1.265 & 1.025 & -19.0 & 1.105 & -12.7 \\
\hline 19 & [bmim] $[\mathrm{Ac}]$ & 1.055 & 1.027 & -2.6 & 1.068 & 1.2 \\
\hline 20 & [emim] $[\mathrm{Ac}]$ & 1.027 & 1.039 & 1.1 & 1.112 & 8.3 \\
\hline 21 & [emim] [BEI] & 1.590 & 1.578 & -0.7 & 1.542 & -3.0 \\
\hline 22 & [bmim] [BEl] & 1.514 & 1.541 & 1.8 & 1.473 & -2.7 \\
\hline 23 & [4MOPY] [BEI] & 1.390 & 1.481 & 6.6 & 1.349 & -3.0 \\
\hline 24 & {$[\mathrm{NH} 221][\mathrm{BEI}]$} & 1.510 & 1.483 & -1.8 & 1.466 & -2.9 \\
\hline
\end{tabular}
calculados y comparados con valores experimentales. (1) Valderrama y Abu-Shark (1989) y (2) Valderrama y Zarricueta (2009).

Tabla 5: Densidad calculada mediante correlaciones generalizadas. 
En otro enfoque, Valderrama et al. (2009) han usado un procedimiento híbrido que incluye el método de contribución de grupos (MCG) como herramienta termodinámica y las redes neuronales artificiales (RNA) como método numérico para correlacionar y predecir la densidad de los líquidos iónicos. La aplicación del método de redes neuronales artificiales requiere de una etapa de entrenamiento en la que la red aprende la relación entre la densidad y la temperatura. Para ello se usaron datos de densidad de 190 líquidos iónicos en función de la temperatura obtenidos de la literatura, teniendo un total de 544 datos. Para discriminar entre los diferentes líquidos iónicos se usaron las siguientes variables: masa molecular $M$ y masa de grupos $M_{G}$ de los grupos que forman la molécula. Para que la red aprendiera más fácilmente los datos de densidad fueron transformados a volumen molar $\left(\mathrm{V}_{\mathrm{m}}\right)$, transformación que permite una relación más lineal entre la variable dependiente (volumen molar) y las variables independientes $\left(T, M, M_{G}\right)$. Para validar la capacidad de aprendizaje de la red se usaron 92 datos no incluidos en la etapa de entrenamiento. Después de analizar varias topologías, la que funciona mejor es (10.15.15.1). La exactitud de los resultados fue verificada determinando las desviaciones relativas y absolutas entre los valores calculados mediante el entrenamiento y los valores experimentales. En la etapa de predicción resultaron valores con desviaciones relativamente bajas, alcanzando un valor promedio de $-0.08 \%$, promedio absoluto de $0.32 \%$ y desviaciones máximas de $2.4 \%$. En predicción, las desviaciones fueron muy similares a las encontradas en la etapa de entrenamiento. La Tabla 6 muestra algunos valores de las desviaciones medias relativas para la densidad predicha por el modelo MCG+RNA propuesto por Valderrama et al. (2009).

Tabla 6: Desviaciones medias relativas de la densidad predicha por el modelo RNA propuesto en Valderrama et al. (2009). La temperatura está en Kelvin y la densidad en $\mathrm{gr} / \mathrm{cm}^{3}$.

\begin{tabular}{|c|c|c|c|c|c|c|c|c|c|}
\hline catión & anión & $T$ & $\rho^{\text {calc }}$ & $\% \Delta \rho$ & catión & anión & $T$ & $\rho^{c a l c}$ & $\% \Delta \rho$ \\
\hline [bdmim] & [PF6] & 323 & 1.198 & -0.36 & [EPY] & [BTI] & 313 & 1.522 & 0.07 \\
\hline [bdmim] & [PF6] & 341 & 1.181 & 0.34 & [EPY] & [BTI] & 318 & 1.517 & 0.08 \\
\hline [bdmim] & [PF6] & 353 & 1.153 & -0.66 & [hmim] & [PF6] & 298 & 1.297 & 0.36 \\
\hline [beim] & [bti] & 295 & 1.409 & 0.36 & [hmim] & [PF6] & 303 & 1.291 & 0.08 \\
\hline [beim] & [NfO] & 295 & 1.399 & -1.99 & [hmim] & [BF4] & 302 & 1.140 & -0.15 \\
\hline [bmim] & [mesy] & 303 & 1.209 & -0.02 & [hmim] & [BF4] & 303 & 1.139 & -0.20 \\
\hline [bmim] & [mesy] & 313 & 1.201 & -0.07 & [hmim] & [bti] & 299 & 1.372 & 0.07 \\
\hline [bmim] & [PF6] & 304 & 1.362 & 0.52 & [hmim] & [bti] & 303 & 1.367 & 0.16 \\
\hline [bmim] & [PF6] & 313 & 1.346 & -0.04 & [hmim] & [BF4] & 306 & 1.136 & -0.28 \\
\hline [bmim] & [PF6] & 310 & 1.350 & 0.03 & [hmim] & [BF4] & 308 & 1.135 & -0.26 \\
\hline [bmim] & [BF4] & 278 & 1.207 & -0.78 & [hmim] & {$[\mathrm{Cl}]$} & 328 & 1.023 & 0.01 \\
\hline [bmim] & [BF4] & 309 & 1.196 & 0.11 & [hmim] & {$[\mathrm{Cl}]$} & 333 & 1.022 & 0.17 \\
\hline [bmim] & [BF4] & 313 & 1.193 & -0.07 & [N1444] & [bti] & 316 & 1.268 & 0.90 \\
\hline [bmim] & {$[\mathrm{Br}]$} & 303 & 1.297 & 0.04 & [N1444] & [bti] & 323 & 1.262 & 0.77 \\
\hline [bmim] & [bti] & 308 & 1.426 & 0.19 & [N1444] & [bti] & 341 & 1.238 & -0.03 \\
\hline [bmim] & [bti] & 323 & 1.410 & 0.46 & [N-bupy] & [BF4] & 313 & 1.203 & -0.20 \\
\hline [bmim] & [bti] & 341 & 1.395 & -0.18 & [N-bupy] & [BF4] & 323 & 1.199 & 0.03 \\
\hline [bmim] & [bti] & 353 & 1.388 & 0.07 & [OHea] & [f] & 298 & 1.178 & -2.18 \\
\hline [bmim] & [dca] & 324 & 1.047 & 0.07 & [OHea] & [f] & 303 & 1.171 & -0.19 \\
\hline [bmim] & [dca] & 356 & 1.026 & -0.02 & [omim] & [BF4] & 317 & 1.084 & -0.62 \\
\hline [bmim] & {$[\mathrm{Br}]$} & 308 & 1.294 & 0.12 & [omim] & [BF4] & 318 & 1.083 & -0.68 \\
\hline [bmim] & [NO3] & 304 & 1.159 & 0.07 & [deim] & [bti] & 295 & 1.473 & 1.44 \\
\hline [bmim] & [NO3] & 323 & 1.145 & 0.17 & [dmim] & [MSO4] & 338 & 1.298 & -0.09 \\
\hline [bmim] & [NO3] & 353 & 1.125 & 0.13 & [edmim] & [bti] & 310 & 1.474 & -0.12 \\
\hline [bmim] & {$[\mathrm{Br}]$} & 318 & 1.287 & 0.00 & [edmim] & [bti] & 323 & 1.468 & 0.17 \\
\hline [bmpyr] & [bti] & 328 & 1.372 & -0.49 & [edmim] & [bti] & 341 & 1.446 & -0.15 \\
\hline [bmpyr] & [bti] & 335 & 1.367 & 0.79 & [emim] & [bti] & 310 & 1.501 & -0.19 \\
\hline [bmpyr] & [bti] & 341 & 1.361 & 0.84 & [emim] & [bti] & 316 & 1.494 & -0.26 \\
\hline [BNM2E] & [bti] & 293 & 1.364 & -0.42 & [omim] & [BF4] & 319 & 1.081 & -0.74 \\
\hline [emim] & [bti] & 323 & 1.488 & -0.32 & [omim] & [BF4] & 322 & 1.078 & -0.88 \\
\hline
\end{tabular}




\section{Presión de Vapor}

Existe limitada información sobre la presión de vapor de los LIs, pero hay áreas de estudio donde se requiere de un valor para la presión de vapor $\mathrm{P}^{v}$, aunque se sabe que es muy baja. Hasta la fecha hay publicados datos de $\mathrm{P}^{\vee}$ para solo cinco Lls: [C2mim][bti], [C4mim][bti], [C6mim][bti], [C8mim][bti] y [bmim][dca]. En nuestro grupo se han analizado ocho modelos, incluyendo los métodos de correlación y los modelos generalizados que ocupan parámetros tales como las propiedades críticas de los fluidos en estudio. Estas propiedades se estiman usando métodos de contribución de grupos como se discutió en una sección anterior.

Los pocos datos de presión de vapor disponibles presentaron algunos problemas para el análisis. Representativo de esta situación es el caso de dos conjuntos de datos para el [C2mim][NTf2] (Emel'yanenko et al., 2007; Zaitsau et al., 2006). Los datos se muestran en la Fig. 2, donde se puede observar una apreciable discrepancia si los datos de un autor se extrapolan hacia la zona de temperatura del otro autor. Situaciones como ésta no permiten un buen análisis de tipo numérico y se debió optar por uno de los conjuntos de datos, basado en conceptos adicionales relacionados con la posible incertidumbre de las mediciones. Para el estudio se seleccionaron los datos de Zaitsau et al. (2006), que fueron obtenidos a más baja temperatura coincidiendo con los demás datos de presión de vapor.

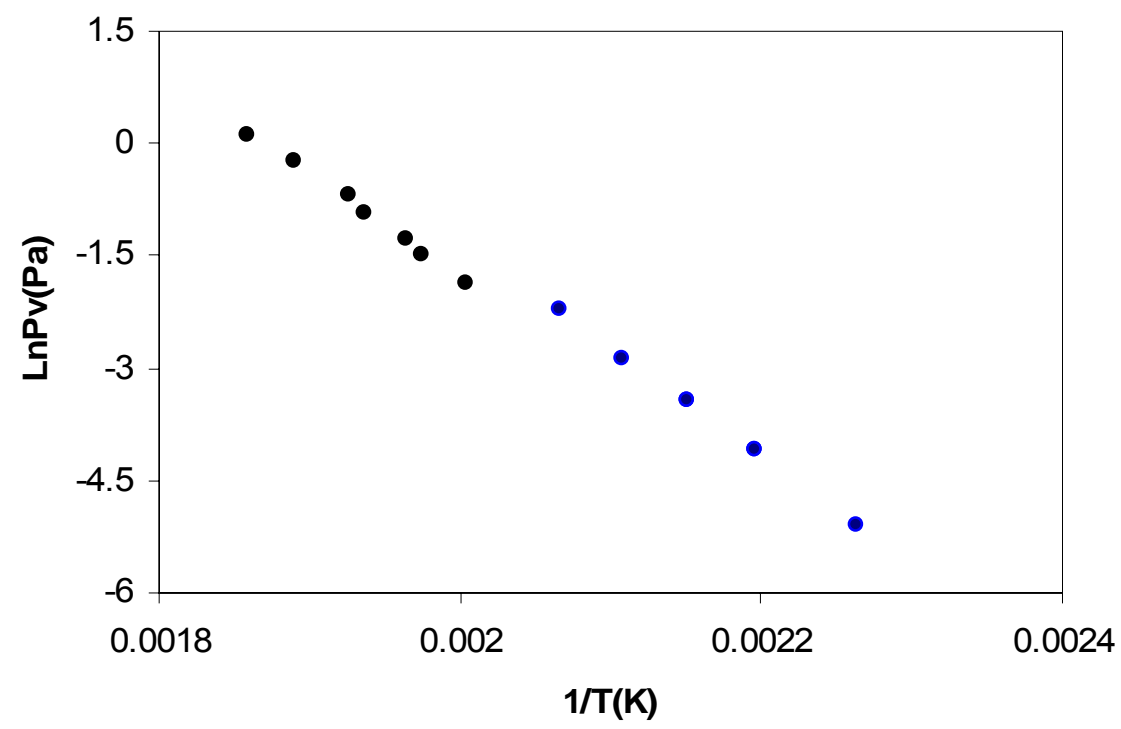

Fig. 2: Presión de vapor de [C2mim][NTf2] de dos autores:

- Emel'yanenko et al. y $\bullet$ Zaitsau et al.

En un primer estudio se consideraron 100 sustancias orgánicas para evaluar la potencialidad de los modelos en la correlación y predicción de la $\mathrm{P}^{\vee}$ y luego se evaluaron los dos mejores modelos para los cinco líquidos iónicos. El estudio se realizó a temperaturas reducidas bajas (T/Tc de 0.3 a 0.5 ), intervalo que corresponde aproximadamente al rango en que existen valores de $P^{\vee}$ para los líquidos iónicos. Una ecuación simple propuesta por Rudkin (1961), y que corresponde a una simplificación y generalización de la ecuación de Antoine dio resultados aceptables.

$\log P^{\vee}=A-B /(T-43)$

El valor 43 para la constante $C$ de la ecuación de Antoine propuesto por Rudkin fue recalculado usando datos de presión de vapor del agua en el intervalo 0.30 a 0.55 de temperatura reducida de 0.3 a 0.55 , obteniéndotese un valor de 38 .

$\log P^{\vee}=A-B /(T-38)$ 
Los valores de esta constante para los cinco líquidos iónicos, fluctúan aproximadamente entre 30 y 40, lo que muestra que el valor 38 corregido parece más adecuado para estimar la presión de vapor de líquidos iónicos. Las constantes $A$ y $B$ pueden ser entonces calculadas usando datos estimados de la temperatura de ebullición normal y las propiedades en el punto crítico, $T_{c}$ y $P_{c}$. Las constantes $A$ y $\mathrm{B}$ son particulares para cada sustancia y fueron determinadas a partir de datos de presión de vapor. La Tabla 7 muestra algunos resultados de la correlación. La desviación media en la correlación de la presión de vapor es $0.6 \%$, la desviación absoluta es $3.2 \%$ y un caso de los analizados solamente da desviación de $17.8 \%$. Valores estimados de $\mathrm{A}$ y $\mathrm{B}$ con $\mathrm{C}=43$ y $\mathrm{C}=38$ son mostrados en la Tabla 8 para algunos LIs del tipo [bti], bis[(trifluoromethyl)sulfonyl]imide.

Otros modelos generalizados más complejos y que hacen uso de las propiedades críticas, tales como Gómez-Nieto-Thodos, Lee-Kesler, y Riedel-Planck-Miller (Reid et al., 1987), no dieron buenos resultados. Esto se puede deber a factores tales como la incertidumbre de los datos experimentales disponibles (se desconoce su grado de exactitud), la temperatura reducida de interés relativamente baja en el caso de los líquidos iónicos ( $\operatorname{Tr}$ entre 0.3 y 0.55 , rango en que las correlaciones disponibles fallan), o las propiedades críticas usadas en estas correlaciones (estimadas mediante métodos de contribución de grupos).

Tabla 7: Valores de las constantes A y B en la ecuación de Antoine-Rudkin, con $\mathrm{P}^{\mathrm{v}}$ en $\mathrm{Pa}$. Los datos tratados son de (1) Zaitsau et al. (2006) y (2) Emel'yanenko et al.(2007)

\begin{tabular}{|c|c|c|c|c|}
\hline Líq. lónico & Rango de T(K) & A & B & Ref. \\
\hline [C2mim][bti] & $441.7-484.2$ & 30.4 & 17209.3 & $(1)$ \\
\hline$[$ C4mim][bti] & $437.8-517.5$ & 30.5 & 17461.3 & $(1)$ \\
\hline$[$ C6mim][bti] & $445.8-493.7$ & 31.0 & 17568.8 & $(1)$ \\
\hline$[$ C8mim][bti] & $455.5-498.2$ & 32.0 & 18343.6 & $(1)$ \\
\hline$[$ bmim][dca] & $448.7-479.6$ & 40.2 & 20593.7 & $(2)$ \\
\hline
\end{tabular}

Tabla 8: Valores de las constantes $A$ y $B$ en la ecuación de Antoine-Rudkin, con $C=43$ y para algunos LIs del tipo bis[(trifluoromethyl)sulfonyl]imide, [bti], con $\mathrm{C}=38$.

\begin{tabular}{|c|c|c|c|c|c|}
\hline \multirow[b]{2}{*}{$N^{0}$} & \multirow[b]{2}{*}{ Ionic Liquid } & \multicolumn{2}{|c|}{ Constantes con $\mathrm{C}=43$} & \multicolumn{2}{|c|}{ Constantes con $\mathrm{C}=38$} \\
\hline & & $A$ & $B$ & $A$ & $B$ \\
\hline 1 & [hpmim] [bti] & 5.33 & 4717.52 & 5.25 & 4675.90 \\
\hline 2 & [nmim] [bti] & 5.48 & 5102.33 & 5.40 & 5058.85 \\
\hline 3 & [pmim] [bti] & 5.19 & 4358.16 & 5.11 & 4318.32 \\
\hline 4 & [prmim] [bti] & 5.07 & 4027.21 & 4.99 & 3989.02 \\
\hline 5 & [prmpy] [bti] & 5.08 & 3979.36 & 5.00 & 3941.00 \\
\hline 6 & [dmeim] [bti] & 5.12 & 4093.35 & 5.04 & 4054.66 \\
\hline 7 & [eomim] [bti] & 5.07 & 4139.60 & 4.99 & 4101.23 \\
\hline 8 & [prmpyr] [bti] & 5.22 & 3612.48 & 5.13 & 3573.31 \\
\hline 9 & [tda] [bti] & 4.64 & 6604.58 & 4.60 & 6565.21 \\
\hline 10 & [thpa] [bti] & 6.20 & 7136.71 & 6.13 & 7084.09 \\
\hline 11 & [dmprim] [bti] & 5.18 & 4257.30 & 5.10 & 4217.79 \\
\hline 12 & [dbim] [bti] & 5.33 & 4717.52 & 5.25 & 4675.90 \\
\hline 13 & [dmim] [bti] & 4.98 & 3726.57 & 4.90 & 3689.87 \\
\hline 14 & [bmpy] [bti] & 5.14 & 4144.03 & 5.06 & 4104.83 \\
\hline 15 & [tpa] [bti] & 6.19 & 5991.88 & 6.11 & 5940.74 \\
\hline
\end{tabular}




\section{Viscosidad}

Se ha explorado el potencial de una Red Neuronal Artificial (RNA) para aprender a relacionar la estructura de la molécula usando el concepto de contribución de grupos y la viscosidad $\mu$ de líquidos iónicos. Los datos publicados por diversos autores presentan diferencias muy notables, que hace difícil el manejo de los datos, en especial cuando se tratan mediante redes neuronales. La Fig. 3 muestra datos encontrados para el [bmim][BF4], caso extremo entre los numerosos datos encontrados. Como no se dispone de técnicas que permitan determinar cuales son los datos correctos y poder eliminar los datos incorrectos, se debieron definir algunos criterios de selección: i) se usaron datos reportados por la NIST (2008); ii) se consideró el comportamiento de la viscosidad con la temperatura; y iii) se analizó la descripción del procedimiento experimental de los autores sobre la exactitud de los datos o las purezas de las muestras. Se seleccionaron 370 datos de viscosidad para 57 líquidos iónicos, la viscosidad varía de 9 a 20883 cP y la temperatura de 278.2 a $348.2 \mathrm{~K}$.

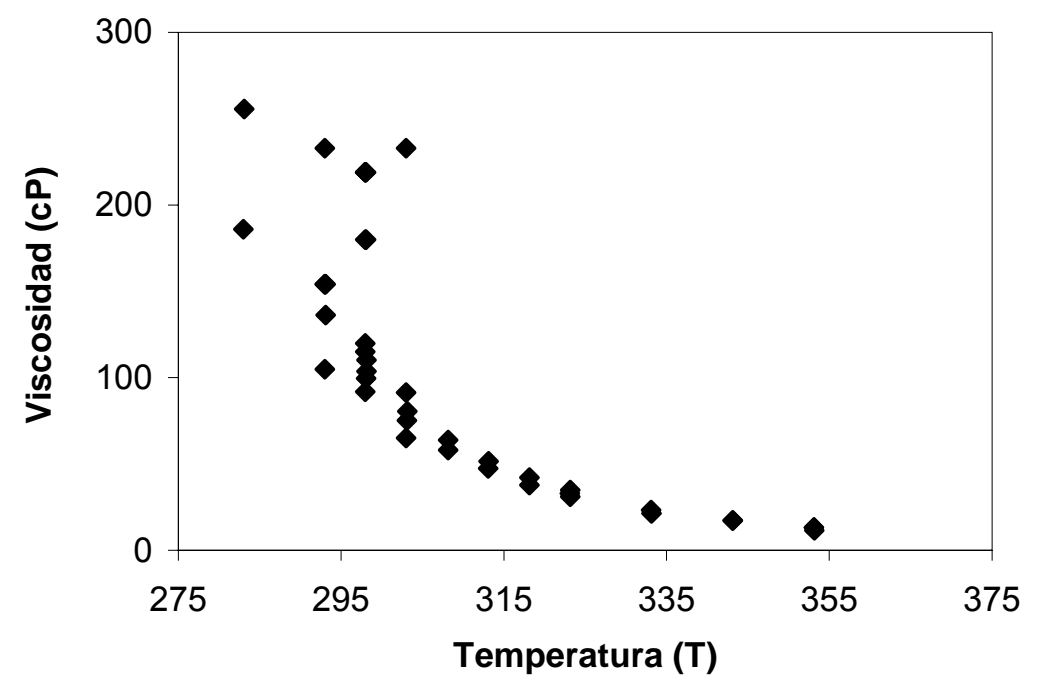

Fig. 3: Viscosidad versus temperatura para [bmim][BF4].

Se desarrollaron rutinas de la RNA usando el software comercial Matlab y se validaron los resultados mediante comparación con los datos disponibles de $\mu$. La red desarrollada se entrenó con datos de $\log \mu$ de 57 líquidos iónicos y un total de 338 puntos. Otros 32 datos se usaron para determinar la capacidad de predicción de la red. Las variables de entrenamiento fueron la temperatura, la densidad, la masa molecular del catión y del anión y el número de grupos en la molécula. Los grupos corresponden a aquellos definidos por los autores para los líquidos iónicos (Valderrama y Robles, 2007a). Después de analizar varias topologías, la que mejor funcionó fue la de estructura 5,15,15,1 (5 neuronas en la capa de entrada, 15 en la cada una de las dos capas internas y una en la de salida). Las desviaciones medias durante el entrenamiento y la predicción fueron inferiores al $3 \%$ y ninguna desviación fue superior al 6\%. Una vez entrenada la red, los parámetros (pesos y ganancias), que determinan el modelo de la relación entre la viscosidad y las variables, quedaron definidos y guardados en un archivo. La Fig. 4 muestra los resultados obtenidos durante la predicción. Se presenta la viscosidad calculada $\mu^{\text {cal }}$ comparada con la experimental $\mu^{\text {lit }}$.

\section{CONCLUSIONES}

Los avances realizados hasta la fecha muestran que los postulados encontrados en la literatura sobre la no aplicabilidad de los métodos usuales de estimación de propiedades que se usan para fluidos orgánicos no pueden ser aplicados a los LIs, no son correctos. Lo resumido en este trabajo indica que los métodos desarrollados para los fluidos moleculares pueden ser extendidos para correlacionar y predecir las propiedades de los LIs con adaptaciones y correcciones apropiadas. Los estudios y avances muestran también que es necesario explorar nuevos métodos para discriminar entre los datos correctos e incorrectos publicados, que las redes neuronales representan una poderosa herramienta numérica de correlación y de predicción y que la contribución de grupos es un 
método apropiado para la predicción de propiedades. En este tema se hace necesario identificar otras propiedades moleculares de los LIs que permitan discriminar entre los isómeros y considerar la distinta complejidad estructural (branching) de los diferentes líquidos iónicos.

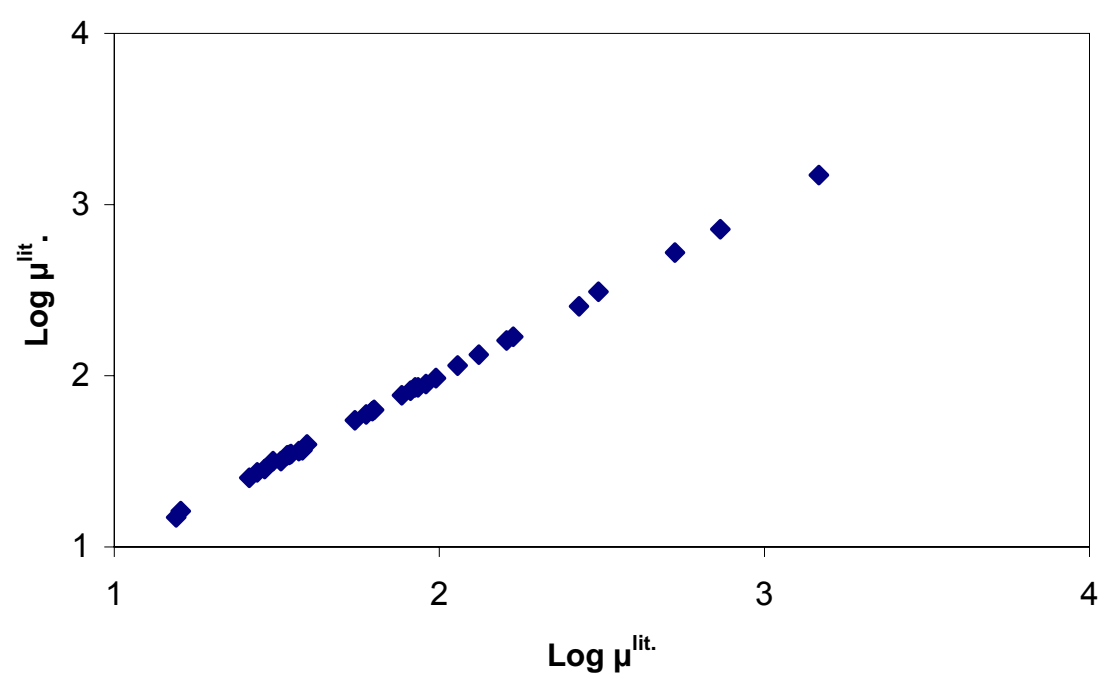

Fig. 4: Resultados preliminares para la viscosidad de los LIs usando redes neuronales.

\section{AGRADECIMIENTOS}

Los autores agradecen el apoyo de Conicyt a través del proyecto FONDECYT 1070025, a la Dirección de Investigación de la Universidad de La Serena-Chile y al Centro de Información Tecnológica de La Serena-Chile.

\section{REFERENCIAS}

Alvarez, V.H. y J.O. Valderrama; A Modified Lydersen-Joback-Reid Method to Estimate the Critical Properties of Biomolecules. Alimentaria: 254, 55-66 (2004).

Emel'yanenko, V., S. Verevkin y A. Heintz; The Gaseous Enthalpy of Formation of the lonic Liquid 1Butyl-3-methylimidazolium Dicyanamide from Combustion Calorimetry, Vapor Pressure Measurements, and Ab Initio Calculations. J. Am. Chem. Soc., 129, 3930-3937 (2007).

GB, GoogleBooks, http://books.google.com/, acceso: Mayo (2009)

ISI, ISI Web of Knowledge, www.isiknowledge.com/, acceso: Mayo (2009)

NIST, National Institute of Standards and Technology, lonic Liquids Database, ILThermo. http://ilthermo.boulder.nist.gov/ILThermo/pureprp.uix.do, access 27 October (2008).

Reid, R.C., J.M. Prausnitz y B.E. Poiling; The Properties of Gases and Liquids. 4th ed., Chap. 7, McGraw Hill Book Co., New York (1987).

Rogers, R.D. y K.R. Seddon; Ionic Liquids-Industrial Applications for Green Chemistry. ACS Symposium Series, vol. 818, American Chemical Society, Washington (2002).

Rudkin, J.; Equation Predicts Vapor Pressures. Chem. Eng.: April 17, 202-203 (1961).

Valderrama, J.O., B. Abu-Shark; Generalized Correlations for the Calculation of Density of Saturated Liquids and Petroleum Fractions. Fluid Phase Equilib.: 51, 87-100 (1989).

Valderrama, J.O. y P.A. Robles; Critical Properties, Normal Boiling Temperatures, and Acentric Factors of Fifty lonic Liquids. Ind. Eng. Chem. Res.: 46, 1338-1344 (2007a). 
Valderrama, J.O. y P.A. Robles; Reply to Comment on Critical Properties, Normal Boiling Temperature, and Acentric Factor of Fifty lonic Liquids. Ind. Eng. Chem. Res.: 46(18), 6063-6064 (2007b).

Valderrama J.O., Sanga W.W. y J.A. Lazzús; Critical Properties, Normal Boiling Temperature, and Acentric Factor of Another 200 lonic Liquids, Ind. Eng. Chem. Res., 47, 1318-1330 (2008a)

Valderrama, J.O., A. Reátegui y W.W. Sanga; Thermodynamic Consistency Test of Vapor-Liquid Equilibrium Data for Mixtures Containing lonic Liquids. Ind. Eng. Chem. Res.: 47, 8416-8422 (2008b).

Valderrama, J.O. y K.A. Zarricueta; A Simple and Generalized Model for Predicting the Density of lonic Liquids. Fluid Phase Equilibria: 275, 145-151 (2009).

Valderrama, J.O. y R.E. Rojas; Critical Properties of lonic Liquids. Revisited. Ind. Eng. Chem. Res., in press (2009).

Valderrama, J.O., A. Reátegui y R.E. Rojas; Density of lonic Liquids using Group Contribution and Artificial Neural Networks. Ind. Eng. Chem. Res.: 3254, 48, 3254-3259 (2009).

Valderrama, J.O., P.A. Robles y R.A. Pizarro; Modelado de Propiedades de Líquidos lónicos para Simulación de Procesos. $8^{\circ}$ Congreso Interamericano CAIP2007, Asunción-Paraguay (2007c).

Valderrama, J.O; Propiedades de Líquidos lónicos Aplicados a Procesos de Separación. Conferencia invitada, $1^{\text {er }}$ Congreso Nacional de Fenómenos de Transferencia, Chiclayo-Perú (c2008).

Valderrama, J.O. y A. Reátegui; Densidad de Líquidos lónicos usando Contribución de Grupos y Redes Neuronales Artificiales. $1^{\text {er }}$ Congreso Nacional de Fenómenos de Transferencia, ChiclayoPerú (c2008).

Valderrama, J.O. y J.A Lazzus; Critical Properties and Vapor Pressure of Twenty Imidazolium based Ionic Liquids used in Extraction Bioprocesses. $2^{\circ}$ Simposio Int. de Biotermodinámica, FrankfurtAlemania (c2008).

Valderrama, J.O., J.A. Lazzus y K. Zarricueta; Density of Imidazolium based Ionic Liquids used in Bioseparation Processes, using Generalized Correlations. $2^{\circ}$ Simposio Int. de Biotermodinámica, Frankfurt-Alemania (c2008).

Valderrama, J.O.; Avances en los Estudios y Aplicaciones de Líquidos lónicos, los Solventes Industriales del Futuro. Congreso Latinoamericano CLICAP. San Rafael-Argentina (c2009)

Valderrama, J.O. y K. Zarricueta; Correlación y Predicción de la Presión de Vapor de Líquidos lónicos. Congreso Latinoamericano CLICAP. San Rafael-Argentina (c2009)

Valderrama, J.O., J. Múñoz y R.E. Rojas; Viscosidad de Líquidos lónicos Usando Redes Neuronales Artificiales y Contribución de Grupos. Congreso Latinoamericano CLICAP. San Rafael-Argentina (c2009)

Valderrama, J.O.; Thermodynamic Consistency Test of Vapor-Liquid Equilibrium Data of Mixtures Ionic Liquid+CO2. Primer Congreso Ibérico sobre Líquidos lónicos, Aveiro-Portugal (c2009a).

Valderrama, J.O.; Advances on the Estimation of Ionic Liquid Properties. Primer Congreso Ibérico sobre Líquidos lónicos, Aveiro-Portugal (c2009b).

Wasserscheid P. y T Welton; Ionic Liquids in Synthesis. Wiley-VCH Verlag GmbH \& Co., Germany (2002)

Welton, T.; Room-temperature lonic Liquids. Solvents for Synthesis and Catalysis. Chem. Rev.: 99, 2071-2083 (1999)

Wypych, G.; Handbook of Solvents. ChemTec Publishing, Toronto-New York (2001).

Zaitsau, D. y otros 6 autores; Experimental Vapor Pressures of 1-Alkyl-3-methylimidazolium Bis(trifluoromethylsulfonyl)imide and a Correlation Scheme for Estimation of Vaporization Enthalpies of lonic Liquids. J. Phys. Chem. A: 110(22), 7303-7306 (2006). 